\title{
Architecture and implementation of IoT system for energy efficient living
}

\author{
Lazar Berbakov, Nikola Tomašević and Marko Batić
}

\begin{abstract}
Recent advances in wireless networking and microelectronic fabrication have enabled a mass production of low-cost computationally capable miniature devices that can be used in different innovative solutions. Since residential sector has been identified as one the most energy demanding, there exists a strong interest in exploitation of connected smart devices with the aim of improving energy efficiency, user comfort and the overall quality of life. The IoT platform that will be presented in this paper aims to reach the aforementioned goals with the combination of smart monitoring and control devices with the advanced energy services capable of analyzing the collected data and providing control actions and suggestions towards the end users via intuitive mobile and web applications.
\end{abstract}

Keywords - energy efficient living, Internet of Things, IoT platform architecture

\section{INTRODUCTION}

Recent advances in wireless networking and microelectronic fabrication have enabled a mass production of low-cost computationally capable miniature devices which can be used in different innovative solutions. Expected growth of human population and steady acceleration of technological advancements will require either an increase of energy production or shifting the general community behavior towards more energy efficient and less wasteful practices. Since residential sector has been identified as one the most energy demanding, there exists a strong interest in exploitation of connected smart devices with the aim of improving energy efficiency, user comfort and the overall quality of life. This challenge was partially addressed during FP7 project Energy Warden [1] where the focus has been put on the optimization of renewable energy technology (RET) deployment in the building domain. On the other hand, during the EPIC-HUB project [2], the aim was to develop a new methodology, an extended architecture and services

This work was partly financed by the European Union (H2020 RESPOND project, Pr. No: 768619, H2020 InBetween project, Pr. No 768776), H2020 LAMBDA project, Pr. No: 809965) and by the Ministry of Science and Technological Development of Republic of Serbia (Pr. No: TR-32010).

Lazar Berbakov, Nikola Tomašević and Marko Batić are with the Mihailo Pupin Institute, University of Belgrade, Volgina 15, 11050 Belgrade, Serbia, (email: name.surname@pupin.rs). able to provide improved Energy Performances to Neighborhoods. This goal was achieved by a combination of pre-existing and new ICT systems with Energy-Hubbased Energy Optimization capabilities.

The platforms that will be developed within the ongoing RESPOND and InBetween project aim to reach the aforementioned goals with the combination of smart monitoring and control devices with the advanced energy services capable of analyzing the collected data and providing control actions and suggestions towards the end users via intuitive mobile and web applications. On one hand, the focus of the RESPOND projects is more towards implementation of collaborative Demand Response (DR) programs that aim to lower the user consumption in times of peak hours, grid overload, etc. In particular, RESPOND will influence the user energy demand by rescheduling some of the loads (e.g. appliances, adjusting heating/cooling, etc.) to suitable time periods (off-peak hours, available renewable energy). The resulting benefit for the end users for participating is reflected in getting the incentives for lowering their consumption. On the other hand, InBetween aims to engage users to identify energy wastes, learn how to conserve energy, motivate them to act and help them to actually carry out energy efficiency practices. Besides, it will deliver affordable solution that brings added value without significant disruption of everyday activities and comfort.

The aim of this document is to present an Internet of Things (IoT) architecture aimed to support energy efficient living. First, the technology deployment scenarios necessary to support the envisioned functionalities are presented, having in mind the constraints such as type of building, occupants' needs, and total investment cost. In particular, the choice of possible deployment models which will be presented, was influenced by a general requirement to integrate different hardware and software components devoted to energy monitoring and automated control at the building/household level. Finally, a preliminary platform architecture focused on IoT cloud functionalities is described.

\section{REFERENCE ARCHITECTURES}

In order to ensure that all the devices communicate using the common messaging format a canonical data model (CDM) shall be defined. In the literature, there exist a number of standards aimed to ensure interoperability in energy management domain. The Energy interoperation presents an information and communication model aimed 
to enable collaborative and transactive use of energy service definitions consistent with the OASIS SOA (Service Oriented Architecture) reference model [3]. The Common Information Model (CIM), a standard developed by the electric power industry that has been adopted by the International Electrotechnical Commission (IEC), aims to enable application software to exchange information about an electrical network [4]. The standard that defines the core packages of the CIM is IEC 61970-301, with a focus on the needs of electricity transmission, where related applications include energy management system, SCADA, planning and optimization.

EEBus SPINE (Smart Premises Interoperable Neutralmessage Exchange) defines a protocol and messages on application layer (ISO-OSI layer 7) that work transparently to the used transport protocol [5]. Any communication technology that provides bi-directional message exchange can be used more or less directly. SPINE supports different use cases that concern control and monitoring of smart appliances like White Goods, HVAC systems and related devices like batteries, etc. Automated Demand Response (ADR) helps system operators to reduce the operating costs of Demand Response (DR) programs [6] while increasing resource reliability. Open Automated Demand Response (OpenADR) is an open and standardized way for electricity providers and system operators to communicate DR signals with each other and with their customers using a common language over any existing IP-based communications network, such as the Internet [7].

\section{IOT PLATFORM USE CASES}

The focus of this section is to elaborate on the possible deployment models for the IoT platform given a general requirement to integrate different hardware and software components devoted to energy monitoring and automated control at the building/household level. The integration problem has been known and investigated for several years and the existing literature has emphasized that the complexity of this integration is severely dependent on the number of different devices employed, existing on-site energy generation and storage etc. In principle, the most widely acknowledged solutions propose architectures that use a data-unifying layer for tackling both syntax and semantic interoperability problem. More precisely, this layer focuses on the abstraction of complexity of lower level, facility related, components and aims to solve existing interoperability challenges through unification and conversion of data coming from these lower software levels. In this way, any format-related issues for pluggable application layers is overcome

On the other hand, when it comes to finding a viable deployment model for such a system, a strong influence is made by the underlying hardware requirements, location of data sources, how data is generated, transported and consumed across the value chain. Moreover, the deployment model also strongly affects the type of business models which can be supported. In other words, it also affects further design of actual software components of the system.

The presented research examines the deployment models from the perspective of the two key stakeholders: the energy utility (both electricity and gas) and the energy end-user, i.e. the customer. For this stakeholder constellation, the three types of deployment models are considered:

- A cloud-based deployment model: which assumes that system components are deployed in a private cloud of an energy provider or energy service company (ESCO). Furthermore, it builds on a fact that both energy monitoring and ICT equipment is under full control of energy and/or service provider and that information about end-users is centrally available. The monitoring equipment located at the end-user is relatively lightweight, e.g. a single smart meter for entire household and does not penetrate into premises of the end-user.

- An extended edge deployment model: system components are distributed between a private cloud governed by the energy/service provider and edge systems deployed at the end-user premises (e.g. energy gateways) and/or distribution network. This deployment model assumes that some of the end-user oriented services can be provided by the edge systems (e.g. energy gateways), thus forwarding only the necessary information to the central system while leaving some simple calculations and decision making (with corresponding alarms and notifications) for the edge systems and components. Energy monitoring, such as smart meters/occupancy/ambience sensing, as well as actuation equipment such as smart plugs/boilers/EVs, can penetrate the end-user premises and offer a richer information and control environment to be locally exploited.

- A hybrid deployment model: this model assumes that not all end-user will have edge system, and corresponding components, deployed at their premises. As a result, some end-users, with limited hardware deployment will only use the cloud-based part of the solution while the others, offering more advanced monitoring and control capabilities, are able to use the system functionalities to the full extent. Such deployment model allows for development of flexible business model and larger market outreach.

Inspired by the previous analysis, the InBetween platform has been designed having in mind different business models that ensure widest possible market coverage and at the same time adapting itself to different user budgets and expectations. In the following sections we will describe three envisioned technology deployment scenarios.

\section{A. Single meter deployment}

The minimum amount of data necessary to get an insight into user's energy consumption behavior can be provided by a single electrical energy metering device. 


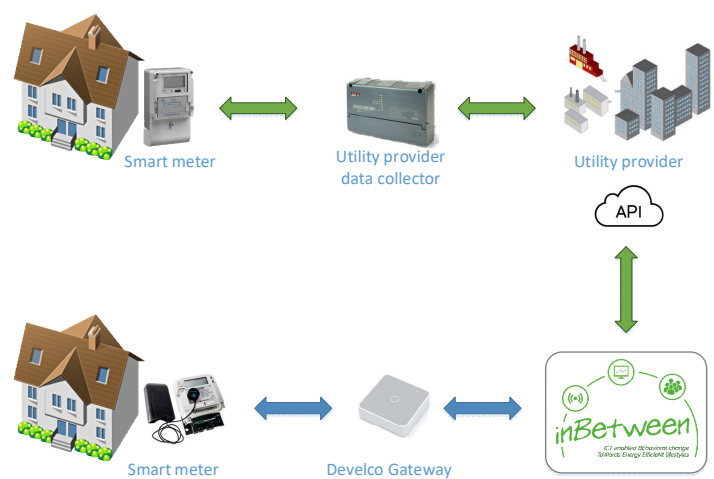

Figure 1. Single meter deployment

Nowadays, the Energy Service Companies (ESCO) usually deploy smart meters that support remote reading of the energy consumption. The main reason behind the usage of smart meters is to lower the operational costs of ESCOs, since previously, authorized personnel needed to visit the user's premises and collect the consumption data manually. Although ESCOs often provide some basic analysis of the collected data on the monthly bill (e.g. energy consumption per month, average consumption, etc.), the full potential of the available data is often left unexploited. If the energy consumption data is collected relatively frequently (on the order of couple of seconds), the machine learning algorithms can be used e.g. to disaggregate the energy consumption per different appliances. In such a way, additional value could be brought to the end user, which could then be able to identify potentially wasteful and costly practices (e.g. using washing machine during peak hours when the energy price is high). This is the exact opportunity that the proposed platform aims to exploit by offering its advanced energy services.

In this regard, we envision two deployment scenarios:

1. Smart meters are property of the ESCO which provides external service API that could be used by IoT platform to acquire energy consumption readings (upper part of Figure 1)

2. Smart meters are deployed by the InBetween project consortium that has full ownership of the data that it collects directly via gateway (lower part of Figure 1).

In the first scenario, which belongs to the group of cloud based deployment models, the project consortium would offer its services to the end users via monthly subscription for the mobile application, in partnership with the ESCO that provides the consumption data via external API. In the second scenario, which belongs to the group of extended edge deployment models, the platform could offer its service subscription directly to the end user without intermediaries, and even for case where non-smart meters are previously available. Nevertheless, in the second scenario there exists an initial cost of equipment installation, that could drawback the potential users, especially if ROI period turns out to be very long.

Since the single meter deployment approach requires no or very little equipment deployment effort on the enduser's side, it allows an easy market entrance. Nevertheless, its main shortcoming when compared to the more comprehensive deployments, which will be described later, is the accuracy of consumption disaggregation that in some cases may lead to erroneous reports and recommendations which would not provide very much value to the end user.

\section{B. Comprehensive energy monitoring and smart sensing deployment}

In this Section, we propose a more comprehensive deployment approach aimed for the end-users that are willing to invest more into smarter monitoring and sensing platform, as shown in Figure 2. As can be seen, this deployment approach mainly relies on the Develco's product [8] portfolio, although similar interoperable devices provided by other manufacturers can be also employed for this purpose.

In Figure 2, we see that there exist different types of sensors that, in addition to power consumption, can also monitor other parameters such as temperature, occupancy, widow/door detector, humidity, etc. The data provided by these additional sensors through a dedicated gateway aim to help the platform to learn more about general user's behavior and its environment and therefore provide more personalized and accurate reports and recommendations for energy saving.

It is important to note that in the comprehensive monitoring deployment scenario, the platform itself is not capable of performing any energy conservation actions automatically. Instead, based on the advanced energy services, the platform is able to predict the energy consumption, schedule the optimal usage of different appliances and detect bad energy efficiency habits. As a result of this analysis, the platform will provide users with a number of recommendations (e.g. close the windows while the HVAC is working, schedule dishwashing machine to work during low pricing period, etc.) aimed to steer their behavior to be more energy efficient and economic.

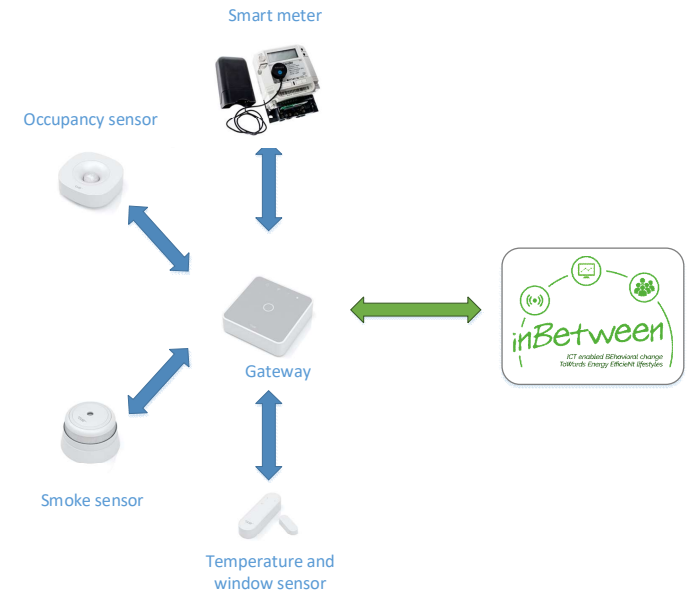

Figure 2. Comprehensive energy monitoring and smart sensing deployment

\section{Comprehensive monitoring, sensing and control deployment}

Building on the capabilities of the previous approach, in this section we propose an extended deployment scenario. 
In Figure 3, we present a comprehensive energy monitoring and deployment approach that is enriched with the capability to perform some energy conservation actions on the user's behalf with previous acknowledgement or even completely automatically. As can be seen, the additional devices, in the form of smart plugs and smart relays, have been added for this purpose. It is important to note that these devices provide only binary control (i.e. on/off) that may not be suitable for all types of appliances found in a household. For instance, a washing machine usually have different types of programs while HVAC system needs more fine grained control regarding temperature set point. To counteract these issues, the platform will support the following control scenarios:

- Manual control, where a user follows recommendations in the case where there is no automatic control capability.

- Semi-automatic control where platform proposes a control action but waits for user action/approval (e.g. although it is daytime, a user might need lights turned on).

- Automatic control where the platform does not require user approval but simply performs an action.

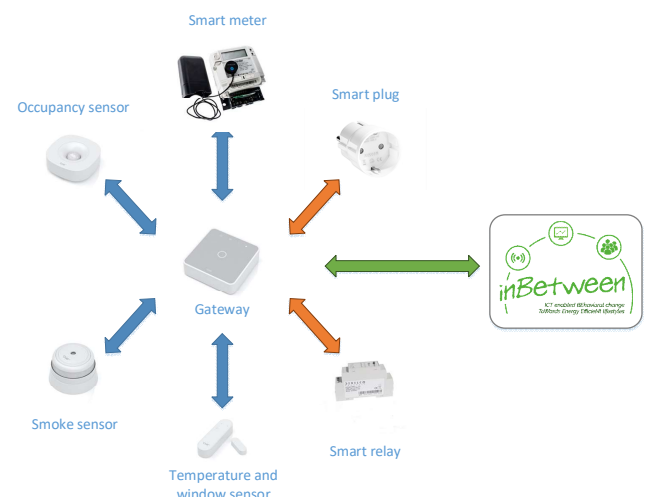

Figure 3. Comprehensive monitoring, sensing and control deployment

\section{IOT PLATFORM ARCHITECTURE}

As can be seen in Figure 4, the cloud based integration layer aims to integrate the advanced energy services developed during the course of RESPOND project with the corresponding monitoring and control devices. It ensures the provisioning and connection of sensors, smart meters and actuators to the network and enable collection, processing and exchange of data among the related components. In particular, In Figure 4 we highlight the InfluxData TICK Stack [9], composed of Telegraf, InfluxDB and Kapacitor. These components can be configured and some tests can be performed in order to check sensor data acquisition, storage and visualization through a testing GUI (Chronograf). InfluxData TICK stack is an open source platform for managing IoT timeseries data at scale, that makes it particularly suitable for the platform development. It allows users to manage metrics, events, and other time-based data.

Time-series databases such as InfluxDB are known to deal with specific workloads and requirements. In particular, they need to ingest a large amount of data points per second, to perform real-time queries across these large data sets in a non-blocking manner, and to down-sample and evict high-precision low-value data. Besides they have to optimize data storage to reduce storage costs and to perform complex time-bound queries to extract meaningful insight from the data. The InfluxData platform is a complete solution able to handle all time series data, from humans, sensors, and machines, seamlessly collecting, storing, visualizing, and turning insight into action.

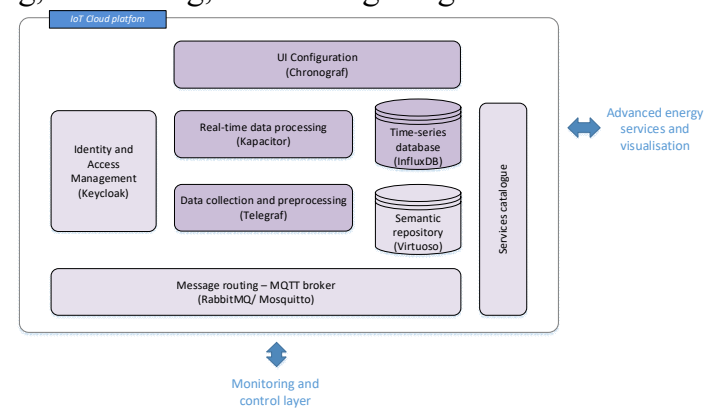

Figure 4. Cloud-based integration layer

The message routing system provides a decoupling of different system functions in the the platform and allows efficient communication between these components following a service oriented paradigm. The messaging exchange system describes the ingestion of inbound data from the field (devices at monitoring and control layer and 3rd Party Services) into the cloud-based system. These data not only include observational data from devices (metrics), but also includes alerting and control data. Having a cloud-based messaging system allows for scalable ingestion and egress (from producers and towards consumers), routing depending on the message type or topic, and differentiation of the producer from the consumer.

\section{CONCLUSIONS}

In this document, we have presented a cloud-based IoT architecture for collection and processing of data collected from distributed sensors with the aim of improving of energy efficiency for smart buildings. In particular, we outlined its main components based on open source TICK stack and other relevant components. Finally, we presented three different technology deployment scenarios that aim to fulfill the technical and functional requirements as well as to support different business models.

\section{REFERENCES}

[1] FP7 project EnergyWarden

[2] FP7 project EPIC-HUB

[3] OASIS Energy Interoperation TC: https://www.oasisopen.org/committees/tc_home.php?wg abbrev=energyinterop

[4] Energy management system application program interface (EMS API): https://webstore.iec.ch/publication/31356

[5] EEBus SPINE: https://www.eebus.org/en/technology/data-model/

[6] Demand response programs https://www.energy.gov/oe/activities/technology-development/gridmodernization-and-smart-grid/demand-response

[7] OpenADR https://www.openadr.org/

[8] Develco products https://www.develcoproducts.com/

[9] Influx data https://www.influxdata.com 